\title{
Hepatoprotective effect of taurine and coenzyme Q10 and their combination against acrylamide-induced oxidative stress in rats
}

\author{
Afaf Abbass Sayed Saleh*, Mona Ismail Shahin, Neveen Atef Kelada \\ Department of Zoology, Faculty of Women for Arts, Science and Education, Ain Shams University, Cairo, Egypt
}

*For correspondence: Email: afafabas1@yahoo.com; Tel: +201111757602

Sent for review: 9 March 2017

Revised accepted: 23 July 2017

\begin{abstract}
Purpose: To clarify the protective effects of Taurine (TA) and Coenzyme Q10 (CoQ10) for mitigation of acrylamide- induced oxidative damage. .

Method: Acrylamide (AA), TA and CoQ10 were administered orally to rats for 2 and 4 weeks. Sixty albino rats of either sex weighing $200 \pm 5$ were randomly divided into five groups; control group, $A A$ group, $A A+T A$ group, $A A+C o Q 10$ group and $A A+T A+C o Q 10$ Group ( 15, 500 and $200 \mathrm{mg} / \mathrm{kg} / \mathrm{day}$ dose, respectively). Hepatoprotection was assessed. The level of hepatic marker enzymes including serum lactate dehydrogenase $(L D H)$, aspartate transaminase (AST) and alanine transaminase $(A L T)$ were evaluated. The proinflammatory cytokines including serum levels of tumor necrosis factor- $\alpha$ (TNF$\alpha)$, interleukin- $1 \beta$ (IL-1 $1 \beta$ ) and interleukin-6(IL-6) were assessed. The levels of reduced glutathione (GSH), malondialdehyde (MDA) and myeloperoxidase (MPO) in liver homogenate were also performed. Results: TA or CoQ10 significantly decreased $(p<0.05)$ the elevation of hepatic markers $(L D H, A S T$ and $A L T$ ) induced by $A A$ in rats. Reduction of serum proinflammatory cytokines (TNF- $\alpha$, IL- $1 \beta$ and IL-6) were also observed compared to AA group, and it was a time-effect relationship. Treatment with TA or CoQ10 also significantly reduced the oxidative stress induced by $A A$ as shown by an increase in GSH level, and reduction of MDA level and MPO activities compared to rats treated with AA alone $(p<0.05)$. The hepatoprotective effect of both TA and COQ10 combination was more efficient than the effect of either of them alone.

Conclusion: The combination of TA and Co Q10 possesses a good potential to inhibit oxidative stress from liver and also exhibits anti-inflammatory activity. Thus, they have the potential to be used to mitigate $A A$ - induced liver injury.
\end{abstract}

Keywords: Taurine, Coenzyme Q10, Acrylamide, Oxidative stress, Biochemical profile, Proinflammatory cytokines

Tropical Journal of Pharmaceutical Research is indexed by Science Citation Index (SciSearch), Scopus, International Pharmaceutical Abstract, Chemical Abstracts, Embase, Index Copernicus, EBSCO, African Index Medicus, JournalSeek, Journal Citation Reports/Science Edition, Directory of Open Access Journals (DOAJ), African Journal Online, Bioline International, Open-J-Gate and Pharmacy Abstracts

\section{INTRODUCTION}

AA (Acrylic amide; prop-2-enamide) represents the chemical formula $\mathrm{C}_{3} \mathrm{H}_{5} \mathrm{NO}$. It is an industrial chemical that exists in monomer and polymer forms [1]. Despite rapid metabolism and excretion following $A A$ exposure, its high reactivity with proteins could be the reason of its toxicity [2]. Besides industrial and laboratory uses, AA formation in foods has its major routes through Maillard reactions of reducing sugars (glucose) with free amino acid (asparagines) under high temperature and low moisture conditions [3]. Maillard reaction is a nonenzymatic browning reaction in foods during baking or frying. Exogenous antioxidants have 
been investigated as being potentially beneficial to decreasing or protecting against oxidative damage [4].

TA is widely distributed in mammalian cells and tissues and has beneficial effects in various physiological and pathological conditions by mainly diminishing production of ROS. Besides, TA addition during food processing significantly inhibits acrylamide formation through directly reacting with $A A$ to promote formation of AA-TA and AA dimmer-TA adducts [5]. Studies have reported that TA can prevent DNA damage at physiological concentrations with hepatoprotective effects through inhibition of extracellular matrix accumulation in experimental liver fibrosis and reverse of liver function tests in rats with hepatic failure. Furthermore, TA enhances the excretion of the hepatotoxic agents, inhibits thioacetamide and acetaminophen bio activation [6].

CoQ10 is an important cellular antioxidant found in all membranes. It is unique among naturally occurring and it is synthesized de novo in animals [7]. Studies have shown that the CoQ10 has free radical-scavenging activities, liver injuryreducing properties and endothelial dysfunction improving [8]. It also has anti-oxidants, antifibrosis and anti- inflammatory effects [9].

Therefore, the present study investigates the hepatoprotective effects of TA and Co Q10 against $A A$-induced hepatotoxicity in rats.

\section{EXPERIMENTAL}

\section{Animals}

The protocol for the present study was approved by the Research Ethics Committee at the Faculty of Science, Ain Shams University, Egypt (RECFS, ref no. 00033). Sixty albino rats of either sex weighing $200 \pm 5 \mathrm{~g}$ were obtained from the Animal House Colony of the National Research Center, Giza, Egypt and allowed to acclimate to the animal facility for 2 weeks prior to the experiment. The rats were housed at $25 \pm 2{ }^{\circ} \mathrm{C}$ with a 12-h light-dark cycle. The animal care and use was performed according to the guidelines of National Research Council (NRC) [10].

\section{Chemical and Drugs dose}

Rats were administered AA of highest purity 99.9 $\%$ at a dose of $15 \mathrm{mg} / \mathrm{kg} /$ day. In the present work, the pharmacological dose of TA and CoQ10 was applied $(500 \mathrm{mg} / \mathrm{kg} /$ day and $200 \mathrm{mg} / \mathrm{kg} /$ day respectively). AA and TA were dissolved in sterile distilled water. CoQ10 was prepared in a $1 \%$ aqueous solution of Tween 80 . All doses were administered orally for two intervals (two and four weeks). All chemicals were purchased from Sigma Chem. Co., St. Louis, MO, USA.

\section{Experimental design and protocol}

The present experiment involced two intervals two and four weeks, respectively. Five groups were formed $(n / 12)$ for the present study (6 rats/ group/ interval). Group I rats received distilled water and served as control group. Group 2 rats received $A A$ (15 mg/kg/day, orally) and served as AA group. Group 3 rats received TA $(500 \mathrm{mg} / \mathrm{kg}$. /day, orally) concomitantly with AA and served as AA+ TA group. Group 4 rats received CoQ10 (200 mg/kg /day, orally) concomitantly with AA and served as AA+ CoQ10 group. Group 5 rats received both TA and CoQ10 concomitantly with $A A$ and served as AA+ TA+ CoQ10 Group. . At the end of each experimental interval (2 and 4 weeks), the animals were decapitated and trunk blood samples were collected for the biochemical investigations.

\section{Determination of serum LDH, AST and ALT}

The activities of serum LDH, AST and ALT were determined spectrophotometrically using an automated analyzer method (Opera Technician Bayer Auto analyzer).

\section{Determination of serum TNF- $\alpha$, IL-1 $\beta$ and IL- 6}

The level of TNF- $\alpha$ was assayed by ELISA (Sandwich Immunoassay Technique). ]. Serum levels of IL- $1 \beta$ and IL- 6 were quantified using enzyme-linked immune-sorbent assay (ELISA) commercial kits specific for rat cytokines. The kits were purchased from IBL-Hamburg Co. (Germany).

\section{Determination of liver GSH, MDA and MPO}

The rats were sacrificed by decapitation; the livers were excised and washed in ice-cold isotonic saline and blotted with a filter paper. Subsequently, the livers were weighed and a portion of the tissue was homogenized in ice-cold $150 \mathrm{mM} \mathrm{KCl}$. The homogenate was centrifuged at $7000 \mathrm{rpm}$ for $15 \mathrm{~min}$ and the resulting supernatant was used for the determination of GSH and MDA levels. Commercial ELISA kit for determination of GSH and MDA was purchased from Oxford Biomedical Research Inc., USA.

MPO activity was assayed by ELISA (Sandwich immunoassay technique) using commercial kit 
(USCN-Life Science INC, China).

\section{Statistical analysis}

Data were evaluated by one-way analysis of variance (ANOVA), followed by the least significant difference (LSD) test using SPSS, version 10 software. $P<0.05$ was considered statistically significant among treatments. All data are expressed as mean \pm standard error (SE).

\section{RESULTS}

The daily oral dose of AA (15 mg/kg) for $2 \& 4$ weeks showed a significant increase $(p<0.05)$ in the enzymatic activities of serum LDH, AST, ALT. In addition, the serum levels of TNF- $\alpha$, IL$1 \beta$ and IL- 6 showed a significant increase $(p<$ 0.05 ) in AA group compared with control group, indicating that the hepatotoxicity was induced by AA. Moreover, depletion of liver GSH was found in rats treated with AA compared with control ( $p$ $<0.05)$. MDA was measured as a marker of lipid peroxidation, and MPO was measured as a marker of neutrophil infiltration. It was found that MDA level and MPO activity were significantly elevated in rats treated with AA compared to control $(p<0.05)$. All adverse alterations increased aggressively with time.

In the current investigation, amelioration effect occurred in the serum LDH, AST and ALT activities of rats group that were treated with 500 $\mathrm{mgTA} / \mathrm{kg}$ day or $200 \mathrm{mg}$ CoQ10/kg /day individually depending on the time of treatment (2 $\& 4$ weeks). (Figure 1). Supplementation of TA or CoQ10 significantly inhibited $(p<0.05)$ the release of serum inflammatory markers and neutrophil infiltration as well as attenuated TNF$\alpha$, IL-1 $\beta$ and IL- 6 levels (Figure 2). In contrast, administration of TA or CoQ10 ameliorated the depletion of intracellular GSH levels, modulated the production of MDA and significantly inhibited and reduced the elevated activities of MPO compared with AA group (Figure 3).

Due to the synergistic effects of both antioxidants (TA \& coq10), all the observed results were significantly reversed $(p<0.05)$. The maximum hepatoprotective effect was observed in the rat groups that were treated with both antioxidants $(\mathrm{AA}+\mathrm{TA}+\mathrm{CoQ} 10)$ depending on the time of supplementation.
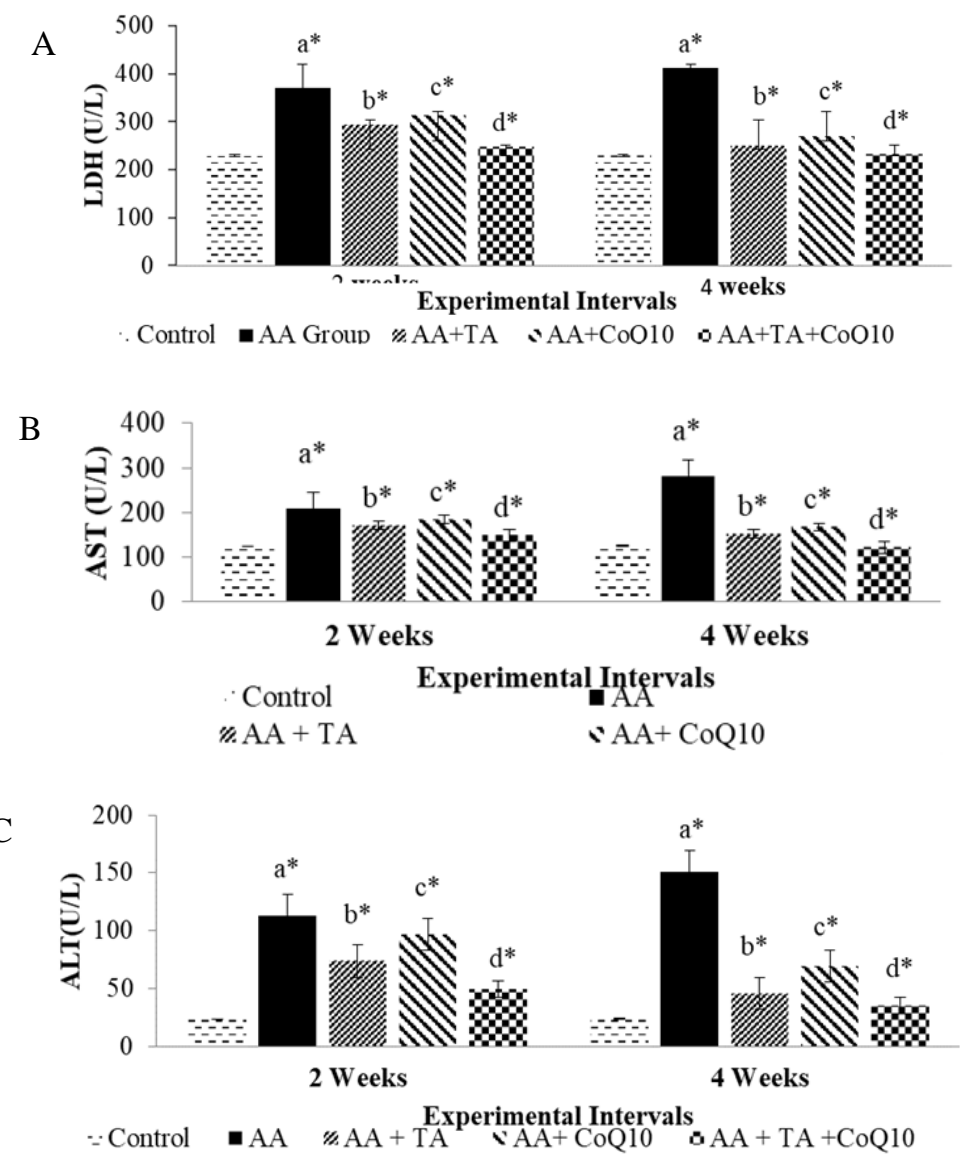

Figure 1: Effect of TA or CoQ10 and their combination on serum LDL (A), AST (B) and ALT (C) activities in AAinduced oxidative stress in rats. Values are expressed as mean $\pm \mathrm{SE}(n=12)$. Asterisk $\left(^{*}\right)$ represents statistical significance; a Compared with control, b, c, d compared with AA Group; * $p<0.05$ 

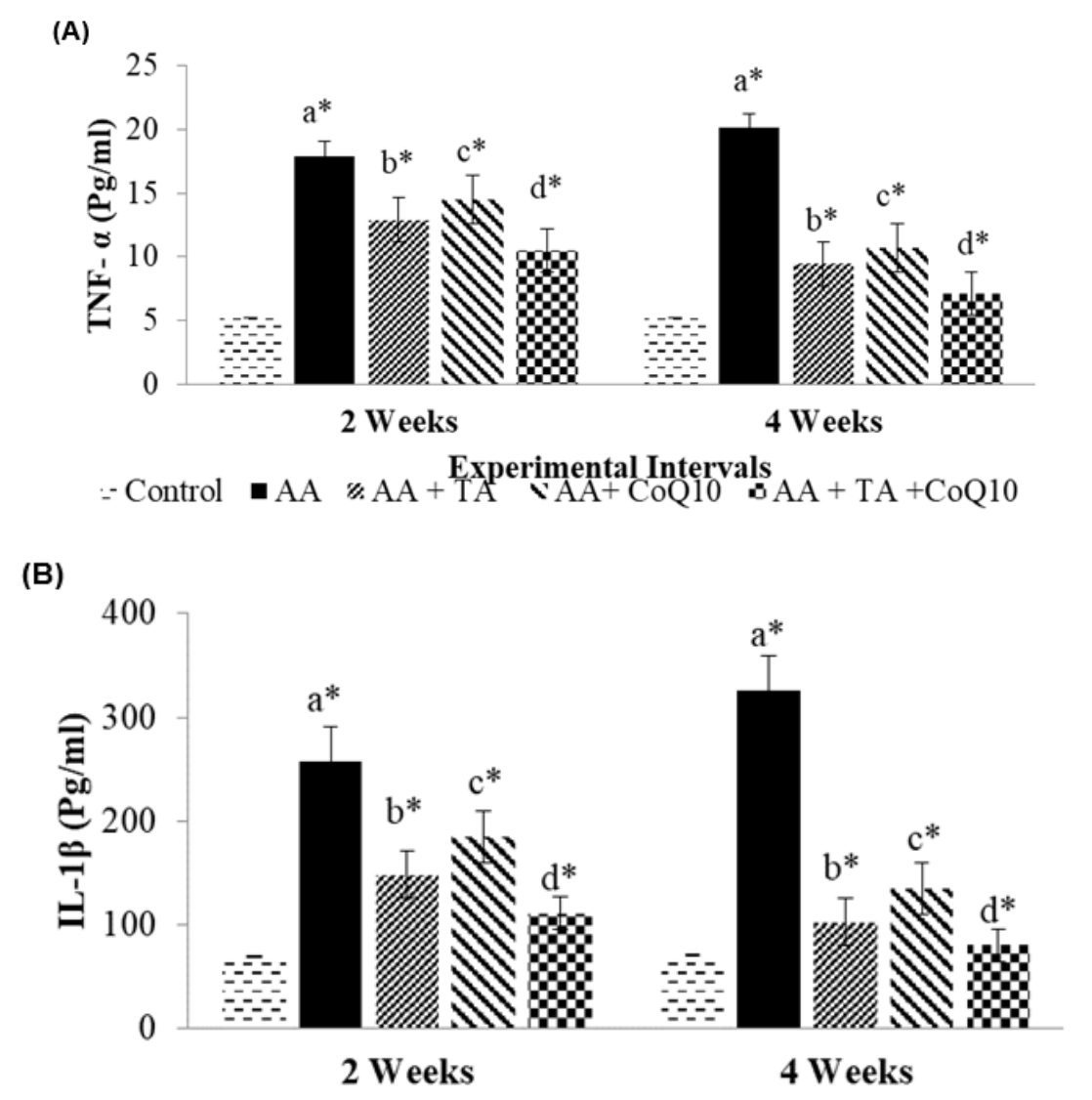

Experimental Intervals

- Control $\square \mathrm{AA} \% \mathrm{AA}+\mathrm{TA}: \mathrm{AA}+\mathrm{CoQ} 10 \approx \mathrm{AA}+\mathrm{TA}+\mathrm{CoQ} 10$

(C)

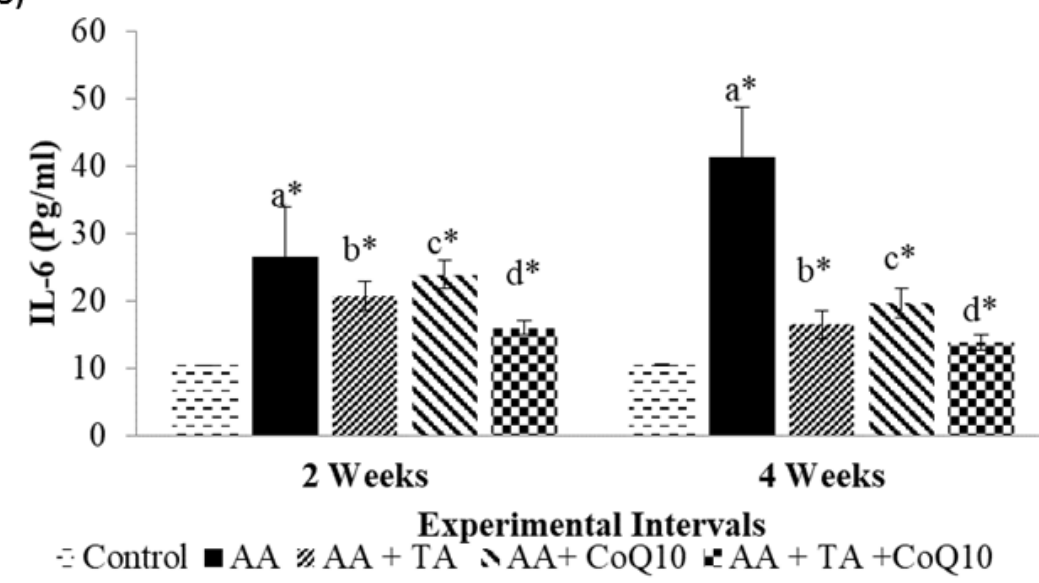

Figure 2: Effects of TA or CoQ10 and their combination on Serum TNF- $\alpha$ (A), IL- $\beta$ (B) and IL-6 (C) levels in AAinduced oxidative stress in rats. Values are expressed as mean $\pm \mathrm{SE}, n=12$. Asterisk $\left({ }^{*}\right)$ represents statistical significance; a Compared with control, b, c, d compared with AA Group; ${ }^{\star} p<0.05$.

\section{DISCUSSION}

AA is a source of toxicities in humans. Its creation takes place during the thermal processing of foods that contain amino acids, mainly asparagine, and reducing sugars. Moreover, long-term oxidative stress creates endogenous acrylamide formation under physiological conditions $\left(37^{\circ} \mathrm{C}, \mathrm{pH} 7.4\right)$ where the majority of AA was conjugated with glutathione while a minimal amount was activated via glycidamide. Both $\mathrm{AA}$ and glycidamide can form covalent adducts with DNA and hemoglobin, as well as induced gene mutation, chromosomal aberration and malignant neoplasm in rodents. Furthermore, AA and its bio-transformed metabolite, glycidamide, are hazardous to different organs such as the liver, 

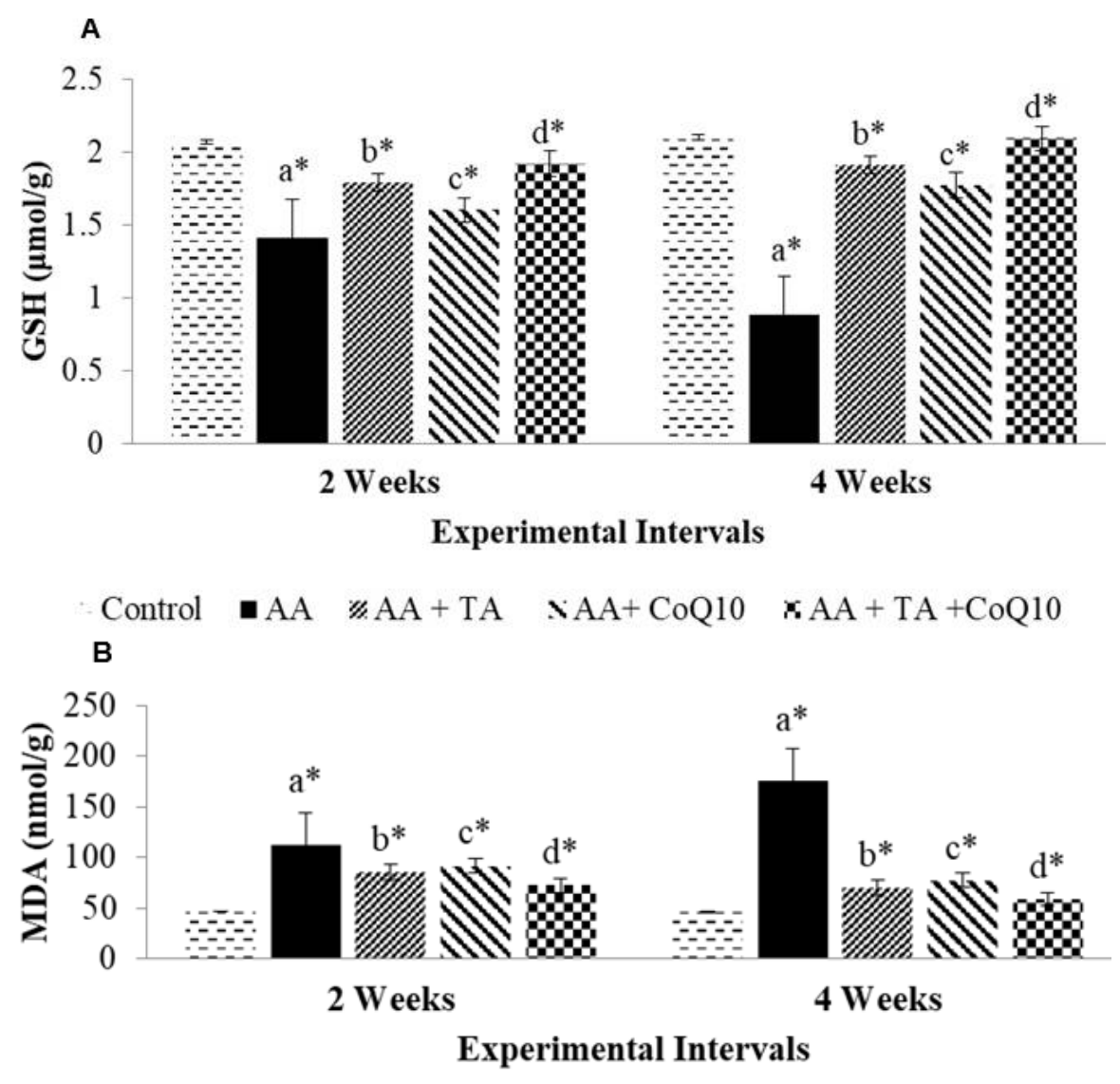

$\therefore$ Control $\because \mathrm{AA} \approx \mathrm{AA}+\mathrm{TA} \backsim \mathrm{AA}+\mathrm{CoQ} 10 \sim \mathrm{AA}+\mathrm{TA}+\mathrm{CoQ} 10$

C

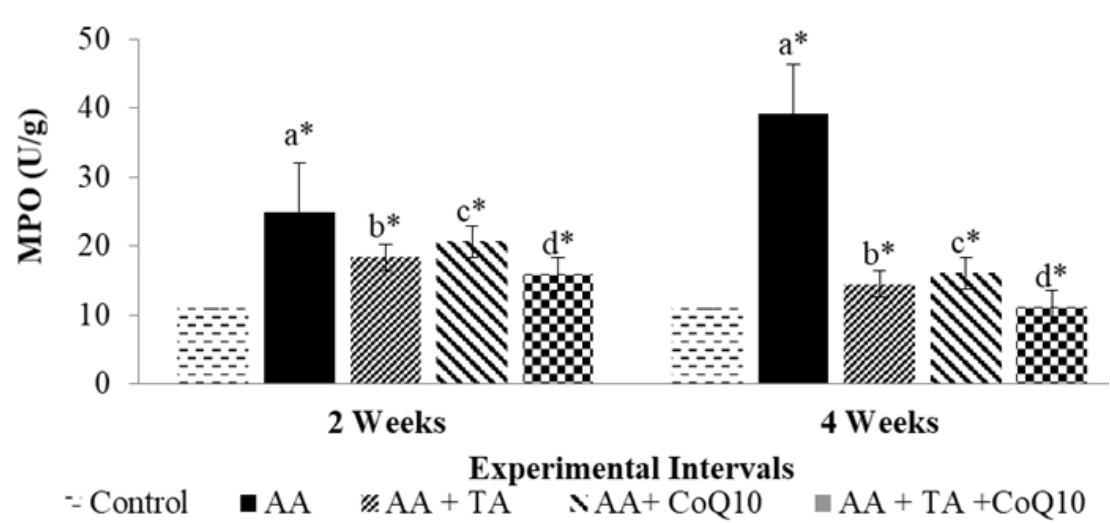

Figure 3: Effect of TA or CoQ10 and their combination on liver GSH (A), MDA (B), and MPO (C) levels in AAinduced oxidative stress in rats. Values are expressed as mean $\pm \mathrm{SE}, n=12$. Asterisk $\left({ }^{*}\right)$ represents statistical significance; a Compared with control b, c, d compared with AA Group; ${ }^{*} p<0.05$

testis and neurons [11].

In the present work, the activities of LDH, ALT and AST in AA group were significantly elevated, suggesting the aggressiveness of $A A$ with time and confirming the advanced permeability of the hepatic membrane after 4 weeks, due to oxidative stress.

Up regulation of pro-inflammatory cytokine expression can also be associated with the generation of free radicals in macrophages at the inflammation stage [12]. The plasma levels of the pro-inflammatory cytokines TNF- $\alpha, \mathrm{IL}-1 \beta$ and IL6 were significantly increased in AA group, revealing hepatic injury; as well as over production of ROS. In addition, significant increase in hepatic MDA level, end products of lipid peroxidation, was also observed in AA group suggesting a relationship between AA exposure and oxidative stress, and is an indicator of hepatic tissue damage. 
GSH is a major intracellular antioxidant as well as an important component in metabolism of many xenobiotic. Recently, it was reported that $A A$ is an oxidative stress inducer. It is efficiently conjugated with GSH to form glutathione-AA adduct that can create cellular oxidative stress. GSH depletion leads to increased lipid Peroxidation and necrosis of hepatocytes. Furthermore, a significant increase in the MPO which removes $\mathrm{H}_{2} \mathrm{O}_{2}$ and catalyzes the formation of toxic hypochlorous acid that leads to neutrophil infiltration and oxidative damage has been reported by Mustafa et al [13]. The present data is consistent with the previous study reporting that in the hepatic Caco-2 intestinal cells, AA induced oxidative stress through depletion of GSH, overproduction of ROS, and induction of apoptosis biomarkers that resulted in cell death [14].

It is interesting to observe that rats treated with TA had a significant improvement of LDH, AST and ALT enzymes, and had a significant suppression of TNF- $\alpha$, IL-1 $\beta$ and IL- 6 ; compared to rats treated with $\mathrm{AA}$ alone. The $\mathrm{GSH}$ content was significantly enhanced, while the degree of liver tissue damage, as measured by the level of MDA and MPO, was also significantly modulated among rats treated with $\mathrm{TA}$; compared to rats treated with AA alone [15]. The present study demonstrates the antioxidant activity of TA against AA-induced hepatotoxicity. TA exerts its antioxidant effect through enhancing membrane stabilization with blocking tissue neutrophil infiltration, balancing oxidant-antioxidant status and regulating the generation of inflammatory mediators.

Supplementation of CoQ10 significantly ameliorated liver function as indicated by $\mathrm{LDH}$, AST and ALT levels and the production of pro inflammatory cytokines, as well as improved hepatic antioxidant defense capacity and attenuated ROS levels. The improvements recorded in all the previous parameters may be explained by the ability of CoQ10 to function as antioxidant, anti-fibrosis and anti-inflammatory agent against AA-induced hepatotoxicity [16].

The current investigation demonstrates that combination of TA and Co Q10 possesses a good potential to inhibit oxidative stress from liver and exhibit anti-inflammatory activity through synergistic properties.

\section{CONCLUSION}

TA in combination with Co Q10 exhibits antioxidant and anti-inflammatory effects through free radical- scavenging activity and creation of
GSH levels, thereby, reducing the liver injury induced by AA.

\section{DECLARATIONS}

\section{Acknowledgement}

The authors gratefully appreciate the providing support by the Faculty of Women for Arts, Science and Education, Ain Shams University, Cairo, Egypt.

\section{Conflict of Interest}

No conflict of interest associated with this work.

\section{Contribution of Authors}

The authors declare that this work was done by the authors named in this article and all liabilities pertaining to claims relating to the content of this article will be borne by them.

\section{Open Access}

This is an Open Access article that uses a funding model which does not charge readers or their institutions for access and distributed under the terms of the Creative Commons Attribution License (http://creativecommons.org/licenses/by/ 4.0) and the Budapest Open Access Initiative (http://www.budapestopenaccessinitiative.org/rea d), which permit unrestricted use, distribution, and reproduction in any medium, provided the original work is properly credited.

\section{REFERENCES}

1. Mojska H, Gielecinska I, Szponar L, Oltarzewski M. Estimation of the dietary acrylamide exposure of the Polish population. Food Chem. Toxicol 2010; 48: 20902096.

2. Krishnakumar $T$, Visvanathan R. Acrylamide in Food Products: A Review. J Food Process Technol 2014; 5: 1-9.

3. Xu Y, Cui B, Ran R. Risk assessment, formation, and mitigation of dietary acrylamide: current status and future prospects. Food Chem Toxicol 2014; 69: 1-12.

4. Kahkeshani $N$, Saeidnia S, Abdollahi M. Role of antioxidants and phytochemicals on acrylamide mitigation from food and reducing its toxicity. J Food Sci Technol 2015; 52: 3169-3186.

5. Hao $R$, Leng $X$, Hao JH. Acrylamide-taurine adducts formation as a key mechanism for taurine's inhibitory effect on acrylamide formation. Int JFood Sci Techno 2011; 46: 1282-1288.

6. Asha KK, Devadasan $K$. Protective effect of taurine on the mitochondria of albino rats induced with fulminant 
hepatic failure. Biomed Prev Nutr 2013; 3: 279-283.

7. Lee BJ, Tseng YF, Yen CH, Lin PT. Effects of coenzyme Q10 supplementation (300 mg/day) on anti-oxidation and anti-inflammation in coronary artery disease patients during statins therapy: a randomized, placebocontrolled trial. Nutr J 2013; 12: 1-9.

8. Laredj LN, Licitra F, Puccio HM. The molecular genetics of coenzyme $Q$ biosynthesis in health and disease. Biochimie 2014; 100: 78-87.

9. Mustafa HN, El Awdan SA, Hegazy GA, Abdel-Jaleel GA. Prophylactic role of coenzyme $Q 10$ and Cynara scolymus $L$ on doxorubicin induced toxicity in rats: Biochemical and immune histochemical study. Indian $J$ Pharmacol2015; 47: 646-656.

10. National Research Council (NRC). Guide for the Care and Use of Laboratory Animals: 8th Ed, 2011. [cited 2013 Apr 3]. Available from: https://grants.nih. gov/grants/olaw/Guide-for-the-Careand-use-oflaboratory-animals.pdf.

11. Manjanatha MG, Guo LW, Shelton SD, Doerge DR. Acrylamide-induced carcinogenicity in mouse lung involves mutagenicity: cll gene mutations in the lung of big blue mice exposed to acrylamide and glycidamide for up to 4 weeks. Environ Mol Mutagen 2015; 56: 446456.
12. Farhangi MA, Alipour B, Jafarvand E, Khoshbaten $M$. Oral coenzyme q10 supplementation in patients with nonalcoholic liver disease: effects on serum vaspin, chemerin, pentraxin 3, insulin resistance and oxidative stress. Arch Med Res 2014; 45: 589-95.

13. Mustafa HN, El Awdan SA, Hegazy GA, Abdel Jaleel GA. Prophylactic role of coenzyme Q10 and Cynara scolymus $L$ on doxorubicin induced toxicity in rats: Biochemical and immune histochemical study. Indian $J$ Pharmacol 2015; 47: 646-656.

14. Rodríguez-Ramiro S, Ramos L, Goya BL, Martín MA. Procyanidin B2 and a coco a polyphenolic extract inhibit acrylamide-induced apoptosis in human Caco-2 cells by preventing oxidative stress and activation of JNK pathway. J Nutr Biochem 2011; 22: 1186-1194.

15. Ali MA, Aly EM, El Awady Al. Effectiveness of selenium on acrylamide toxicity to retina. Int $J$ Ophthalmol 2014; 7: 614-620.

16. Sanoobar M, Eghtesadi S, Azimi A, Khalili M, Khodadi B, Jazayeri S, Gohari MR, Aryaeian N. Coenzyme Q10 supplementation ameliorates inflammatory markers in patients with multiple sclerosis: a double blind, placebo, controlled randomized clinical trial. Nutritional Neuroscience 2015; 18: 169-176 